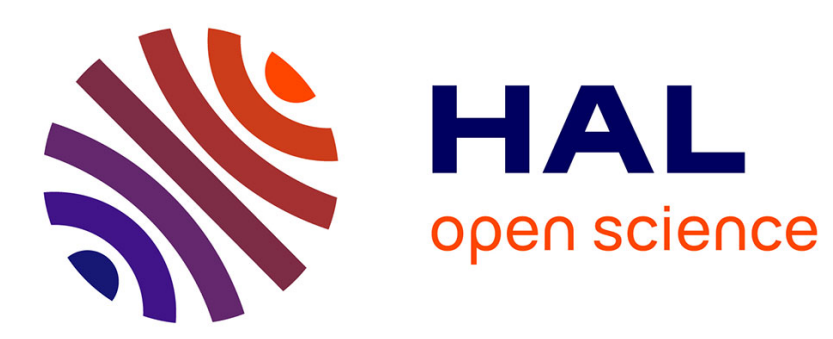

\title{
Reaction Mechanisms in Shocked, Intercalated Graphite and Boron Nitride
}

\author{
R. Bardo
}

\section{To cite this version:}

R. Bardo. Reaction Mechanisms in Shocked, Intercalated Graphite and Boron Nitride. Journal de Physique IV Proceedings, 1995, 05 (C4), pp.C4-561-C4-568. 10.1051/jp4:1995445 . jpa-00253751

\section{HAL Id: jpa-00253751 https://hal.science/jpa-00253751}

Submitted on 1 Jan 1995

HAL is a multi-disciplinary open access archive for the deposit and dissemination of scientific research documents, whether they are published or not. The documents may come from teaching and research institutions in France or abroad, or from public or private research centers.
L'archive ouverte pluridisciplinaire HAL, est destinée au dépôt et à la diffusion de documents scientifiques de niveau recherche, publiés ou non, émanant des établissements d'enseignement et de recherche français ou étrangers, des laboratoires publics ou privés. 


\title{
Reaction Mechanisms in Shocked, Intercalated Graphite and Boron Nitride
}

\author{
R.D. Bardo \\ Naval Surface Warfare Center, 10901 New Hampshire Avenue, Silver Spring, MD 20903, U.S.A.
}

\begin{abstract}
The traditional ways of developing increasingly energetic materials usually lead to an increase in shock and impact sensitivities. It is, therefore, of practical and theoretical importance to design model, highly-energetic polycrystalline systems which will clearly indicate, at the molecular level, the interplay between the shock-induced reaction mechanisms and the associated excited lattice states. Theoretical and experimental studies indicate that such systems may possibly be constructed from special materials such as high-quality pyrolytic, layered graphite and hexagonal boron nitride (BN) crystals. Although each layer of graphite and $\mathrm{BN}$ is one of the most stable structures in nature, intercalation of the crystals with various oxidizing agents can yield energetic systems with the desired properties. As an example, intercalation with $\mathrm{HNO}_{3}$ gives crystals of density $2.20 \mathrm{~g} / \mathrm{cc}$. The optimal positioning of the $\mathrm{HNO}_{3}$ molecules between the $\mathrm{BN}$ layers allows the rapid formation of $\mathrm{B}_{2} \mathrm{O}_{3}$ in a single step with a large release of energy. A possible triggering mechanism is the shock-induced, partial $\mathrm{sp}^{3}$ hybridization of the layers as a result of kink band formation.
\end{abstract}

\section{INTRODUCTION}

New energetic compounds continue to be synthesized which give ever-improving fragment acceleration and blast pressure, but often showing a concomitant increase in sensitivity. Since the traditional "trial and error" methods used in the scale-up of the new materials from laboratory to test conditions continue to show conflicting performance and sensitivity results, it would be of practical and theoretical importance to be able to design, from the ground up, a model highlyenergetic system which clearly exhibits the needed insensitivity and performance at all stages of development. It is, therefore, the purpose of this paper to indicate the feasibility of such a program by exploiting the current understanding of the nature of the molecular-level processes governing the behavior of energetic materials at high pressures and temperatures.

Benchmark experiments exist which show profound differences in the way homogeneous and heterogeneous materials initiate under shock. In the case of sustained shock pulse initiation of homogeneous liquid nitromethane (NM), 1 which is free of discontinuities of any kind, fast reaction appears to begin close to the driver plate interface after an induction time of about $10.6 \mathrm{sec}$. No light is emitted directly behind the initiating shock, which at 60 kbar propagates $8 \mathrm{~mm}$ before initiation begins at the interface. The absence of light emission indicates no appreciable excitation of electronic states with lifetimes $\leq 10^{-6} \mathrm{sec}$. Similar behavior is observed in molten trinitrotoluene (TNT) 1 as well as in single crystals of pentacrythritol tetranitrate (PETN), 1 cyclotrimethylene trinitramine $(\mathrm{RDX})^{2}$ and cyclotetramethylene tetranitramine (HMX). 2 On the other hand, sustained shock pulse initiation of heterogeneous NM containing air and oxygen bubbles and of polycrystalline solids gives rise to initiation close to the initiating shock with negligible induction times. ${ }^{1}$ In all of these materials, sensitivity is a function of the defect structure and heterogeneous nature of the medium. 
More recently, directional shock sensitivity has been discovered in single crystals of PETN by Dick, et al. ${ }^{3}$ who showed that, in the chosen geometry, initiation is difficult to achieve along the crystal direction of greatest slip. Although existing crystals of shock-insensitive, "graphitic" triaminotrinitrobenzene (TATB) are too small to be reliably studied with the techniques used for PETN (crystal size of $1 \mathrm{~cm}$ ), it is expected that larger specimens would show similar, but more pronounced behavior. In PETN, all three orthogonal directions have van der Waals bonding, whereas the layered structure of TATB has such bonding only in one direction and strong intralayer hydrogen bonding. 4 Other explosives with similarly-pronounced slip systems would be expected to show directional sensitivity properties as well. It should be emphasized that while the above research pertains to the structure of single crystals, an understanding of the effects of polycrystalline interactions are crucial in the final analysis of shock sensitivity. Toward this goal, van der Steen, et al. 5 have, for example, determined that crystalline shapes significantly affect sensitivity.

The present paper discusses the design of high-performance crystals which clearly exhibit directional shock sensitivity properties along specific crystal axes and yet which clearly approach the insensitivity of homogeneous energetics along the other axes. Such materials are being constructed from layered pyrolytic boron nitride $(\mathrm{BN})$ crystals which are intercalated with $\mathrm{HNO}_{3}$ molecules.

\section{INTERCALATION OF BN}

Intercalation compounds are formed by insertion of a guest chemical species - an intercalate between layers in a host material. Because of its simple structure, high-quality pyrolytic graphite is most often the preferred choice of host lattice for purposes of enhancing its electrical conductivity and chemical reactivity. 6 Another simple lattice is pyrolytic $B N$, the crystal structure of which is closely related to that of graphite and being built of hexagonal layers of the same kind, but arranged so that atoms of one layer lie vertically above those in the layers below.

Although each layer in graphite or $\mathrm{BN}$ is one of the most stable structures in nature, intercalation of crystals with various oxidizing agents can yield explosive systems with the desired properties. In the case of $\mathrm{BN}$, highly-energetic reactions are possible with formation of $\mathrm{B}_{2} \mathrm{O}_{3}$

$\left(\Delta \mathrm{H}_{\mathrm{f}}=-303 \mathrm{kcal} / \mathrm{mol}\right)$. Under ordinary laboratory conditions, the molecules of intercalate enter the host crystal by exploiting the weak binding energy $(1.5 \mathrm{kcal} / \mathrm{mol}$ or $0.065 \mathrm{ev})$ between the layers and increasing the interlayer spacing. 6

Intercalation proceeds by charge transfer from the host $\mathrm{BN}$ layers to the oxidizer molecules, causing bonding within the layers of intercalate. This intercalate-intercalate bonding may be stronger than the intercalate-host bonding, resulting in a large thermal expansion of the intercalate layer relative to that of the host layer, which exhibits almost no thermal expansion. For the oxidizer or acceptor compounds, the intercalate layer becomes negatively charged by extracting electrons predominantly from the host bounding layers. Thus, these host layers have a high concentration of holes, causing the Fermi level to fall and the corresponding cylindrical Fermi surface to shrink.

It is interesting to note here that intercalation of "graphitic" TATB would likely produce a much less stable structure, since the transfer of charge would be small and localized in the vicinity of the carbon rings. As a consequence, the intercalate-intercalate bonding would be weaker than that found in the extended structures with uniform bonding in the host layers.

While ordinary procedures of doping give random distributions of guest species, intercalation produces a highly-ordered structure. The resulting process of staging gives a mechanism for controlled variation of the physical properties of the compounds. Stage $\mathrm{m}$ compounds have $\mathrm{m}$ graphite or BN layers between successive layers of intercalate. For maximum intercalation, $m=1$, and the theoretical maximum density (TMD) of the crystal falls in the range $1.80 \leq \mathrm{TMD} \leq 2.25 \mathrm{~g} / \mathrm{cm}^{3}$ for the simple molecular intercalates. In this case, the host and intercalate layers alternate so that the structure is uniform and "homogeneous". In the case of planar $\mathrm{NO}_{3}$, calculation with the MNDO method ${ }^{7}$ gives an equilibrium, horizontal stacking of the $\mathrm{BN}$ and $\mathrm{NO}_{3}$ layers, as shown in Figure 1 for two $\mathrm{NO}_{3}$ molecules and two truncated structures

\section{$\mathrm{B}_{k} \mathrm{~N}_{1} \mathrm{H}_{13}$. An estimation of the density $\rho$ for the corresponding $m=1$ system gives}

$\rho=1.80 \mathrm{~g} / \mathrm{cm}^{3}$, which is much smaller than the value of $2.25 \mathrm{~g} / \mathrm{cm}^{3}$ for hexagonal $\mathrm{BN}$. On the other hand, we find that intercalation of $B N$ in fuming nitric acid gives an $m=1$ density of

$2.20 \mathrm{~g} / \mathrm{cm}^{3}$. The only structure which has this density is the one of optimal vertical packing of $\mathrm{HNO}_{3}$ molecules shown in Figures $2 \mathrm{a}$ and $2 \mathrm{~b}$. Calculations with MNDO give the positions of the hydrogen-bonded $\mathrm{HNO}_{3}$ intercalate indicated in Figure $2 \mathrm{~b}$. The entire structure appears to be 
stabilized by hydrogen bonding and by the electrostatic forces between the net positive and negative charges on the B and O atoms, respectively. Further calculations of the mechanism of intercalation may indicate that the $\mathrm{HNO}_{3}$ molecules initially enter the $\mathrm{BN}$ lattice horizontally followed by their rotation to the vertical orientation in order to allow macroscopic stabilization of the entire host/intercalate structure.
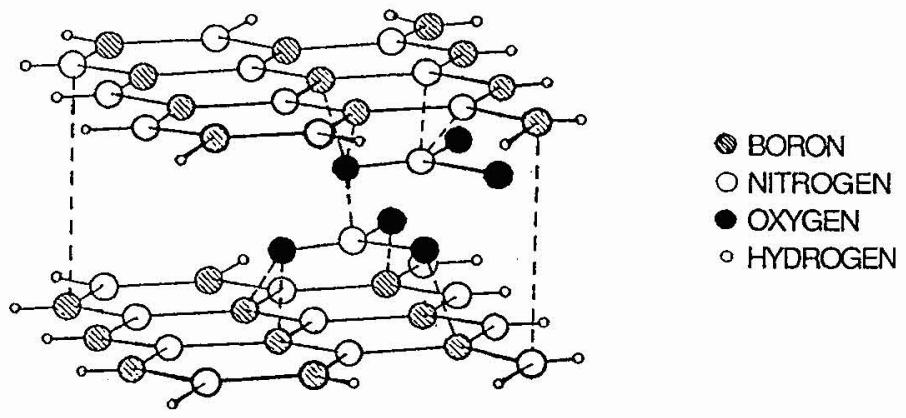

Figure 1. Calculated equilibrium geometry of two $\mathrm{HNO}_{3}$ molecules between two layers of $\mathrm{B}_{\mathrm{k}} \mathrm{N}_{1} \mathrm{H}_{13}$.

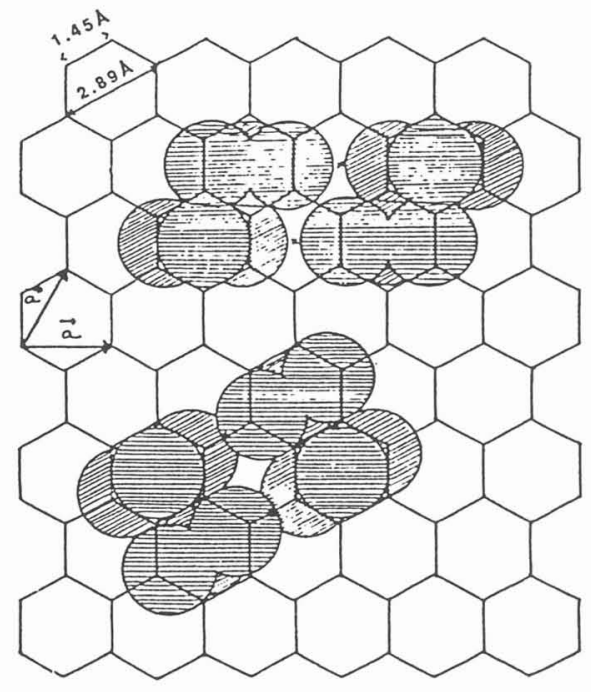

(a)

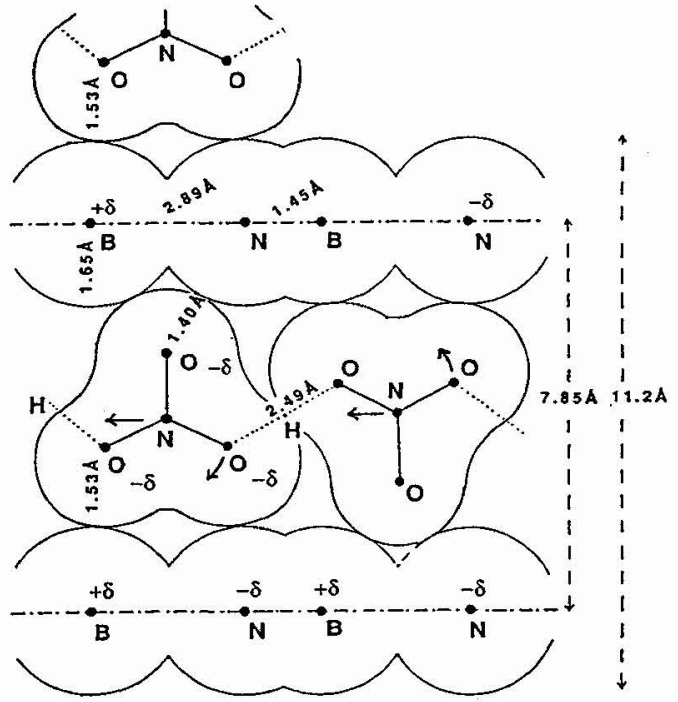

(b)

Figure 2. (a) Two arrangements of $\mathrm{HNO}_{3}$ molecules perpendicular to the (001) plane of $\mathrm{BN}$. Side-by-side configuration of molecules in lower part of Figure $2 \mathrm{a}$ is the most stable one. (b) Side view of the lowest energy arrangement. 
Since slip in the layered materials must occur without breakage of the strong B-N bonds, basal dislocations must be present to allow deformation of the hexagons. 8 For stage 1 compounds, maximum slip along the glide planes of dislocations is possible, since the Burgers vectors corresponding to the active basal dislocations of the layers are parallel to the basal plane. 8 In this case, there is minimal slip perpendicular to the basal plane. These dislocations, which split into two partials, have associated with them shear, acoustic modes which are soft (low frequency $\omega)$. For stages $m>1$, regions or galleries of intercalate species are formed between adjacent host layers, resulting in strain of the graphite or BN layers. In graphite the presence of a layer of intercalate causes the two adjacent host layers to undergo relative shear to bring them into eclipsed stacking. The presence of a dislocation at a boundary of a gallery has, then, a Burgers vector with basal and perpendicular components. 8 The presence of the latter component corresponds to an edge dislocation, allowing slip to zls s occur perpendicular to the basal plane.

\section{TIMES TO IGNITION IN SHOCKED, INTERCALATED BN}

A theory 9 has been developed by the author which provides a formal framework and guide for the analysis of shock ignition in layered materials. This theory describes the interrelationship among bimolecular chemical reactions and processes for vibrational energy transfer between the crystal lattice and its molecules. Chemical reaction occurs only after sufficient energy is transferred for activation of the molecules. In many cases, this is the slow or rate-determining step, the characteristic time $t_{p v}$ for which is given by

$$
t_{p v}=\hbar \sqrt{\rho_{a} \rho_{o}}
$$

where $\hbar, \rho_{a}$, and $\rho_{o}$ are, respectively, Planck's constant and the densities-of-state for the acoustic and optical lattice modes. As indicated in Reference $9, \rho_{\mathrm{a}}$ and $\rho_{\mathrm{o}}$ may be calculated from the general expression

$$
\rho=\left(\frac{2}{\pi n}\right)^{1 / 2} \frac{(1-1 / 12 n) \lambda}{h(\nu\rangle(1+\eta)}\left[\left(1+\frac{\eta}{2}\right)\left(1+\frac{2}{\eta}\right)^{n / 2}\right]^{n}
$$

where $\mathbf{n}$ is the number of vibrational degrees of freedom, $\langle\boldsymbol{v}\rangle$ is the average of the $\mathrm{n}$ frequencies $v_{i}$, and $\lambda$ and $\eta$ are defined by the equations

$$
\begin{aligned}
\lambda^{-1} & =\prod_{i}\left(\nu_{i} /\langle\nu\rangle\right), \\
\eta & =E l E_{0} .
\end{aligned}
$$

In Equation (2c), $\mathrm{E}$ is the internal energy interval and $\mathrm{E}_{0}$ is the zero-point energy. Equations (2a)(2c) apply to both harmonic and anharmonic vibrations.

Calculation of the energy transfer times $t_{p v}$ indicates the important role of slip in the initiation of PETN, as interpreted earlier by Dick, et al. 3 Since excitation of the higher-energy optical modes of the lattice is crucial to ignition, ${ }^{9}$ preferential excitation of the long-wavelength acoustic modes corresponding to slip can, by Equation (1), give times $t_{p v}$ which are too slow for the given dimensions of the crystal.

The importance of $t_{p v}$ may be seen in context with other processes occurring in the system which also have characteristic times. These processes and their corresponding rate coefficients pertain to (1) energy transfer from the host lattice into the intercalation molecules $k_{p v}=t_{p v} v^{-1}$, (2) energy transfer from the molecules back into the host lattice $k_{v p}$, and (3) bimolecular reaction $\mathrm{k}_{\mathrm{b}}$ between host and intercalate, which combine to give the total rate coefficient 9

$$
k_{\text {tot }}=k_{p v} k_{b} /\left(k_{v p}+k_{b}\right)
$$


If most of the shock energy is dissipated into the low-frequency acoustic vibrations corresponding to the direction of greatest slip, little reaction is generated so that $k_{v p} \gg k_{b}$ in Equation (3). If, then, $k_{\text {tot }}{ }^{-1}>t_{r}$, where $t_{r}$ is the time for arrival of rarefaction waves, any reaction is quenched and no ignition is possible, since reduction of the shock pressure $P_{s}$ below a critical value inhibits the important bimolecular reactions. For the single $1 \mathrm{~cm}$ PETN crystals used in Reference 3, calculation shows that $k_{\text {tot }}{ }^{-1} \cong 10^{-5} \mathrm{sec}$ for $10 \leq \mathrm{P}_{\mathrm{s}} \leq 80 \mathrm{kbar}$, and $\mathrm{t}_{\mathrm{r}} \cong 10^{-6} \mathrm{sec}$ for shocks along crystal direction $\langle 100\rangle$. On the other hand, if $k_{\text {tot }}{ }^{-1} \leq t_{r}\left(t_{\text {tot }} \geq t_{r}\right)$, ignition is possible. This is the case for orientation $<001>$ where slip is minimized and $k_{b} \gg k_{v p}$, so that $k_{\text {tot }}{ }^{-1}=t_{p v}$. Here, calculation shows that $\mathrm{k}_{\text {tot }^{-1}}<10-10 \mathrm{~s} \mathrm{c}$ for $10 \leq \mathrm{P}_{\mathrm{s}} \leq 80 \mathrm{kbar}$. These results for PETN are portrayed in Figure 3. It is emphasized here that it is the slow, rate-determining step, identified with $t_{p v}$, which ultimately determines the ability of the material to ignite at certain critical pressures and temperatures.

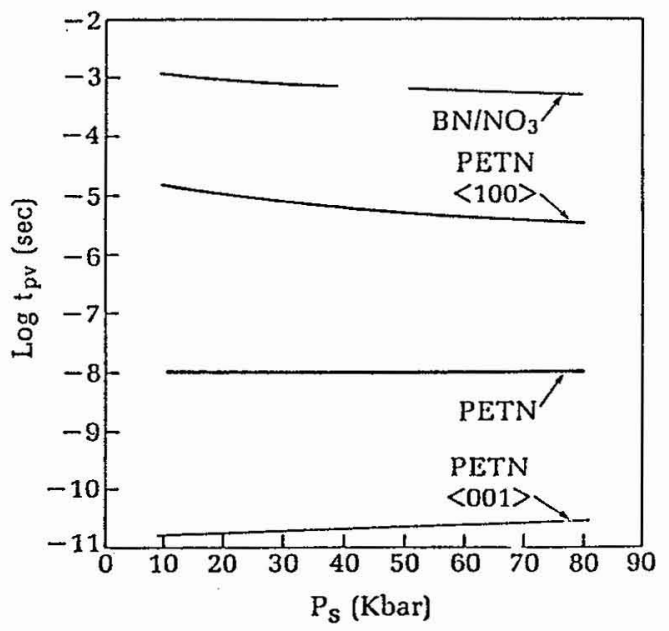

Figure 3. Comparison of shock pressure dependence of $t_{\mathrm{pv}}$ for acoustic and optical modes along various crystal directions in $\mathrm{BN} / \mathrm{NO}_{3}$ and PETN. The curve labelled PETN corresponds to the geometric mean of the densities-of-state, Eq.(1), for directions $<001>$ and $<100>$. Upper two curves for $\mathrm{BN} / \mathrm{NO}_{3}$ and PETN pertain to maximum slip.

For initiation to occur in layered crystals, shocks must have a sufficiently large component of stress $\mathrm{P}_{\mathrm{cs}}$ along the c-axis of the unit cell. At some critical shock stress $\mathrm{P}_{\mathrm{cs}}$, excitation of the initiating optical modes along the c-axis results in bimolecular reaction between the host and intercalate layers, and $t_{p v}<t_{r}$. For the $\mathrm{BN} / \mathrm{NO}_{3}$ system depicted in Figure $2 b$, an estimate of $t_{p v}$ is readily obtained from Equations (1) and (2) if the following reasonable assumptions are made:

(1) initiation along the c-axis is effectively one-dimensional and (2) $P_{c s} \approx 130 \mathrm{kbar}$, which approximates the known minimal pressure required to cause significant distortion of the BN or graphite planes to form the cubic structures. $10 \mathrm{It}$ is assumed here that the lower bound to the optical mode quantum hw along the c-axis of BN is about $70 \mathrm{~cm}^{-1}$, which is the value for highquality graphite. 11 Also, the ratio $\lambda \cong 1$ in Equation (2b) and $E \approx 5 \times 10^{9} \mathrm{ergs} / \mathrm{g}$ at $P_{c s}=130 \mathrm{kbar}, 12$ so that in Equation $(2 \mathrm{c}), \eta \approx 14.3$, since $E_{0} \approx 35 \mathrm{~cm}^{-1}$. The number of atoms per unit cell in the $\mathrm{BN} / \mathrm{NO}_{3}$ structure at stage 1 is 10 , and the corresponding number of optical modes $\mathrm{n}$ for the one-dimensional problem is 9 . Substitution of all of these values into Equation (2a) gives, then, $\rho_{0} \leq 1.2 \times 10^{24} / \mathrm{erg}$. Accordingly, since $\mathrm{n}=1$ for the acoustic mode along the c-axis, substitution of the remaining parameters above into Equation (2a) gives

$\rho_{\mathrm{a}} \leq 7.8 \times 10^{13} / \mathrm{erg}$. Thus, from Equation (1), $\mathrm{t}_{\mathrm{pv}} \leq 10^{-8} \mathrm{sec}$, which is much shorter than $t_{r} \cong 10^{-6}$ sec for centimeter-scale crystals. 
The effects of sustained shocks of $130 \mathrm{kbar}$ at increasing angles from the c-axis are, to good approximation, two-dimensional in the crystal, corresponding to slip of the host and intercalate planes past each other, because of the relatively weak host/intercalate interaction under shock conditions. In this case, $\mathbf{n}=2$ and 18 for the acoustic and optical modes, respectively. Substitution of these values as well as the ones above for $\hbar \omega, \lambda$, and $\eta$ into Equation (2a) gives $\rho_{\mathrm{a}} \leq 1.1 \times 1015 / \mathrm{erg}$ and $\rho_{\mathrm{o}} \leq 4.7 \times 1035 / \mathrm{erg}$, and from Equation (1), $t_{\mathrm{pv}} \leq 0.02 \mathrm{sec}$. Here then, $t_{p v} \gg t_{r}$, and initiation is unlikely. For lower pressures, additional calculations indicate that $t_{p v} \approx 10^{-3} \mathrm{sec}$ in the range of $10 \leq P_{c s} \leq 80 \mathrm{kbar}$. In Figure 3 , these times are seen to be longer than those for PETN, indicating the much greater insensitivity of $\mathrm{BN} / \mathrm{NO}_{3}$ crystals of comparable size.

A similar analysis of TATB, the planar molecules of which form a graphitic structure, is expected to yield times in the range $10^{-5} \leq t_{\mathrm{pv}} \leq 10^{-3}$. This is a result of the rigidity ( $r$ ) of the slip planes for the shock pressures indicated, where $r$ has the order PETN $<$ TATB $<B N / N_{3}$.

\section{KINK BANDS AND REACTION}

In Figure $2 \mathrm{~b}$, the arrows indicate how the $\mathrm{NO}_{3}$ molecules adjust their positions under compression along the $\mathrm{c}$-axis to form chains of nearest-neighbor $\mathrm{B}$ and $\mathrm{O}$ atoms. Ignition along the c-axis can then result from reaction between these atoms to form $\mathrm{B}_{2} \mathrm{O}_{3}(\mathrm{O}=\mathrm{B}-\mathrm{O}-\mathrm{B}=\mathrm{O})$ in a single step with high exothermicity. As an example, reaction of $\mathrm{BN}$ and $\mathrm{HNO}_{3}$ for the stage 1 stoichiometry $(\mathrm{BN})_{2} \mathrm{HNO}_{3}$ would yield $1.57 \mathrm{kcal} / \mathrm{g}$ (energy density of $3.45 \mathrm{kcal} / \mathrm{cm}^{3}$ ), $6 \%$ higher than HMX. Here, the reaction is not diffusion-limited, in contrast to the situation for most known multi-component energetic materials. Thus, the activation energy in $\mathrm{k}_{\mathbf{b}}$ of Equation (3) will have the same significance of a homogeneous reaction. The activation parameters have not yet been evaluated for the formation of $\mathrm{B}_{2} \mathrm{O}_{3}$.

For stage-1 $\mathrm{BN} / \mathrm{HNO}_{3}$ crystals, deformation by slip and the dynamic distortions (vibrations) considered in Section 3 and in Reference 13 do not significantly affect the activation energy. Twinning, on the other hand, which results in the static buckling of the BN planes indicated in Figure 4, can play a role. In contrast fo slip, twinning results from buckling of the BN planes, as indicated in Figure 4 for the basal plane. This source of strain acts as a source of energy for reaction, and so aids the chemical process. The corresponding strain energy, which arises for $\mathrm{sp}^{2}$ to $\mathrm{sp}^{3}$ rehybridization, is approximated by $\Delta \mathrm{E} \approx 17 \mathrm{kcal} / \mathrm{mol}(1420 \mathrm{cal} / \mathrm{g})$.

For many layers of $\mathrm{BN}$, twinning results in kink bands, each of which is defined as the region between two walls of dislocations of opposite sign, 14,15 as shown in Figure 5. Kink bands have been observed in graphite. ${ }^{8}$ The shape of the kink band and its size are determined by the integrity of the $\mathrm{BN}$ or graphite planes. The size is approximated by the equilibrium condition 15

$$
\frac{w}{L} \ln \left(\frac{L}{w}\right)=\pi(1-\sigma) \theta\left(\frac{\tau_{x y}}{C_{44}}\right)
$$

where $\sigma$ is the Poisson ratio $\approx 1 / 3, \theta$ is the angle of kinking $\leq 20^{\circ}$, and the ratio of the shear stress component $\tau_{\mathrm{xy}}$ to the shear modulus $\mathrm{C}_{44}$ is $(\cos \alpha \cos \beta) \mathrm{P}_{\text {uniaxial }} / \mathrm{C}_{44}$, which is approximately 1/40. The magnitude of $\mathrm{C}_{44}$ for $\mathrm{BN}$ is larger than $10 \mathrm{kbar}$ at high stress levels. Equation (4) is obtained by minimizing

$$
\frac{\mathrm{U}_{0}}{\mathrm{~N}}=\frac{\mathrm{C}_{44}}{2 \pi(1-\sigma)}\left[(w \theta)^{2} \ln \left(\frac{\mathrm{L}}{w}\right)-b L \theta \ln \theta\right]
$$

where the Burgers vector magnitude $b \approx 1.45 \AA$. From Equation (4), $w / L \approx 0.35$ and $U_{0} \approx$ $613 \mathrm{cal} / \mathrm{g}$ for $\mathrm{BN}$. At $\mathrm{P}_{\text {uniaxial }}=300 \mathrm{kbar}$, for example, $(1 / 2) \mathrm{P} \Delta \mathrm{V} \approx 830 \mathrm{cal} / \mathrm{g}$ from the $\mathrm{BN}$ 
Hugoniot. The total change in internal energy under shock loading is, therefore, $(1 / 2) \mathrm{P} \Delta \mathrm{V}+\mathrm{U}_{0} \approx$ $1400 \mathrm{cal} / \mathrm{g}$. This elastic strain energy, which approximates the rehybridization energy of $1420 \mathrm{cal} / \mathrm{g}$ given above, is stored in the kink bands.

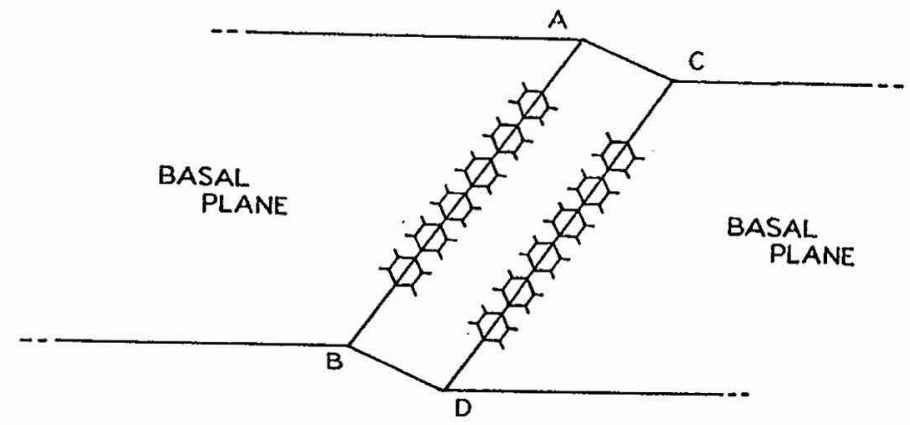

Figure 4. Twin band consisting of two twin boundaries at $\mathrm{AB}$ and $\mathrm{CD}$ in the basal plane.

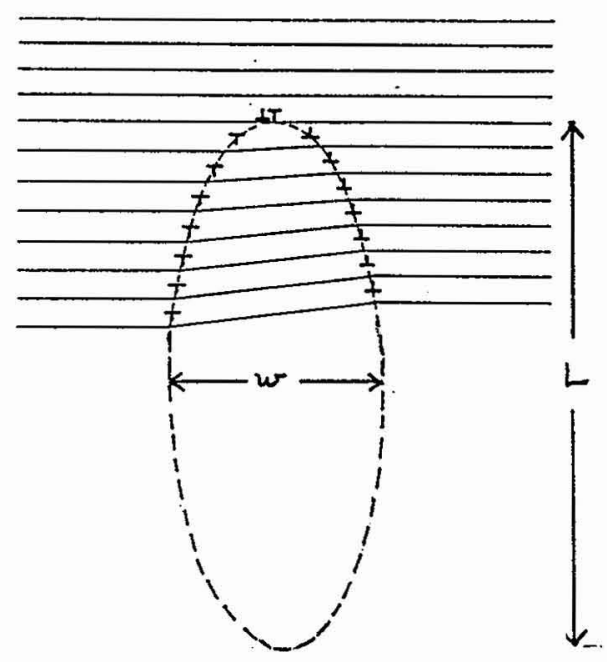

Figure 5. Kink band consisting of twin boundaries in graphite or hexagonal BN. 


\section{SUMMARY}

The important criteria for optimal directional sensitivity in intercalated, layered $\mathrm{BN}$ materials are that (1) they must be stage 1 or close to it; (2) the interplanar activation energies for reaction between BN and the intercalate layers must be less than those for reaction within each intercalate layer; and (3) the important interlayer reactions must be bimolecular and exothermic. Criterion (1) addresses the requirement of minimal slip along the crystal axis perpendicular to the basal plane. In this case, $t_{p v} \| \gg t_{p v}$, for the parallel and perpendicular directions. Criterion (2) addresses the need to eliminate or minimize reaction in the intercalate layer as a result of energy transfer from the gliding host layers caused by shocks not normal to the basal plane. This requires the careful selection of intercalate. Criterion (3) guarantees that the reactions will occur in times which are short compared to arrival of rarefaction waves caused by shocks. These reactions occur with activation energies that are much lower than those in unimolecular processes. The rates of these reactions given by $\mathrm{k}_{\mathrm{b}}$ can be sufficiently fast at the pressures and temperatures of interest in detonations, ${ }^{9}$ so that "initiating" energy transfer from the BN lattice into the intercalate molecules is the slow step. Localization of strain energy in kink bands aids the subsequent chemical processes. Reaction occurs at the location of the kink bands. Together, these criteria help to guarantee that the most likely direction of shock initiation is normal or near-normal to the basal plane.

\section{ACKNOWLEDGMENT}

Special thanks are extended to Prof.R. Armstrong for helpful discussions.

\section{REFERENCES}

[1] Campbell A.W., Davis W.C., and Travis J.R., Phys. Fluids 4, 498 (1961).

[2] Campbell A.W. and Travis J.R., in Proceedings of the Eighth Symposium (Int.) on Detonation, NSWC MP 86-194, 15-19 Aug 1985, p.1057.

[3] Dick J.J., Mulford R.N., Spencer W.J., Pettit D.R., Garcia E., and Shaw D.W., J. Appl. Phys. 70, 3572 (1991).

[4] Cady H.B. and Larson A.C., Acta Cryst. 18, 485 (1965).

[5] van der Steen A.C., Verbeek H.J., and Meulenbrugge J.J., in Proceedings of the Ninth Symposium (Int.) on Detonation, Vol. 1, OCNR 113291-7, 28 Aug-1 Sep 1989, p. 83.

[6] Bartlett N.and McQuillan B.W., in Intercalation Chemistry, edited by Whittingham M.S. and Jacobson A.J. (Academic Press, New York, 1982), pp. 19-53.

[7] Dewar M.J.S., Thiel W.J.J., J. Am. Chem. Soc. 99, 4899, 4907 (1977);

Stewart J.J.P. MOPAC-MNDO Program in QCPE 1986, 18, p. 455.

[8] Amelinckx S., Delavignette P., and Heerschap M., in Chemistry and Physics of Carbon, Vol. 1, edited by Walker P.L. (Marcel Dekker, Inc., New York,1965), pp. 1-71.

[9] Bardo R.D., Int. J. Quantum Chem. S20, 455 (1986); Bardo R.D., in Proceedings of the Ninth Symposium (Int.) on Detonation, Vol. 1, OCNR 113291-7, 28 Aug-1 Sep 1989, p. 235.

[10] Coleburn N.L. and Forbes J.W., J. Chem. Phys. 48, 555 (1968).

[11] Green J.F., Bolsaitis P., and Spain I.L., J. Phys. Chem. Solids 34, 1927 (1973).

[12] Rice M.H., McQueen R.J., and Walsh J.M., in Solid State Physics, edited by Seitz F. and Turnbull D. (Academic Press Inc., New York, 1958), pp. 1-63.

[13] Bardo R.D., in Structure and Properties of Energetic Materials, MRS Vol. 296, edited by Liebenberg D., Armstrong, R. and Gilman J., (Mat. Res. Soc., May, 1993), p. 186.

[14] Freise E.J. and Kelly A., Proc. Roy. Soc. (London) Ser. A264, 269(1961).

[15] Frank F.C. and Stroh A.N., Proc. Phys. Soc. (London) 65, 811(1952). 